\title{
QUÍMICA VERDE: UN COMPROMISO VINCULANTE ENTRE NATURALEZA, ACADEMIA Y SOCIEDAD
}

\author{
Renato Contreras
}




\section{RENATO CONTRERAS}

Es Profesor Titular del Departamento de Química de la Facultad de Ciencias de la Universidad de Chile. Doctor en Física Molecular Teórica por la Universidad de Paris VI-Sorbonne (1982). Es además miembro del Grupo de Química Teórica de la Facultad de Ciencias y Director del Centro Interdisciplinario de Líquidos Iónicos (CILIS). 


\section{QUÍMICA VERDE: UN COMPROMISO VINCULANTE ENTRE NATURALEZA, ACADEMIA Y SOCIEDAD}

\section{INTRODUCCIÓN}

La Química Verde (QV, Anastas \& Warner 1991) es una forma innovadora de enfrentar y remediar los aspectos negativos relacionados con la industria química como un ente generador de beneficios, minimizando el impacto negativo en el entorno, incluyendo todos sus aspectos: toxicidad, energía y efecto invernadero. En este sentido, la QV difiere de la Química Ambiental (QA) en sus fundamentos más esenciales. Mientras la QA es una disciplina orientada a remediar los desechos y daños producidos por la actividad industrial, la QV está orientada a prevenir y minimizar dicho impacto ("El mejor chef no es el que más limpia, sino el que menos ensucia"). El mecanismo que utiliza la QV puede ser resumido en una palabra clave, a saber, el diseño de procesos químicos alternativos que privilegian la preservación del entorno por sobre los beneficios económicos de la actividad industrial (Cann \& Connely, 2000). Por definición, la palabra diseño implica inmediatamente que la QV es una actividad esencialmente multidisciplinaria, en el sentido que experimento y teoría deben actuar en forma simultánea y sinérgica para lograr el objetivo principal de obtener procesos alternativos para obtener un mismo producto, pero en condiciones que preservan la calidad del entorno humano en toda su dimensión. Esta manera de actuar responsablemente ha sido definida en base a una serie de 12 postulados (Anastas \& Warner, 1991) que se enumeran a continuación con una breve definición.

\section{POSTULADOS DE LA QUÍMICA VERDE}

\section{Prevención}

Es preferible anticipar el daño al entorno transferido como desecho que tratar de eliminarlo después de producido.

\section{Economía atómica}

La ruta sintética recomendada debe ser diseñada de manera de maximizar la incorporación de los materiales de partida (reactantes) en el producto final. 


\section{Síntesis químicas menos peligrosas}

En la medida de lo posible, los métodos sintéticos deberían ser diseñados en orden a usar y producir susstancias que poseen una muy baja o ninguna actividad tóxica para el ser humano y el entorno.

\section{Diseño de compuestos menos agresivos con el entorno}

Los productos químicos deben ser diseñados para cumplir su función deseada, minimizando su toxicidad.

\section{Uso de solventes y dispositivos auxiliares solo cuando son inevitables}

El uso de sustancias auxiliares en síntesis, tales como solventes o dispositivos de separación (membranas) debiera evitarse, en lo posible, o ser inocuos cuando sea inevitable su uso.

\section{Diseño sintético enfatizando la eficiencia en el consumo de energía}

Los requerimientos de energía para todo proceso químico deben ser diseñados haciendo un balance racional entre el impacto económico y el ambiental. El límite ideal en las condiciones de síntesis es realizar el proceso a presión y temperatura ambiente.

\section{Uso de materias primas renovables}

Los materiales de partida o materia prima deben ser renovables para evitar agotarlas, siempre que sea técnica y económicamente factible.

\section{Reducción de productos derivados o secundarios no deseados}

En lo posible, evitar en un paso sintético el uso de químicos auxiliares, como por ejemplo grupos protectores de reactividad en regiones específicas del material de partida, debido a que tales procedimientos incorporan reactivos adicionales que puedan permanecer como desechos al final del proceso.

\section{Catálisis}

Agentes catalíticos, en lo posible selectivos, son una alternativa superior a reactivos estequiométricos.

\section{Diseño sintético privilegiando una degradación óptima del producto}

Los productos de un proceso químico pueden y deben ser diseñados de modo que al final de su función no persistan en el ambiente. 


\section{Análisis en tiempo real para la prevención de contaminantes}

Es necesario desarrollar técnicas analíticas apropiadas para mantener el control de la producción de contaminantes in situ durante el proceso previo a la formación de sustancias peligrosas.

\section{Diseño de rutas sintéticas inherentemente inocuas para la prevención de accidentes}

La selección de procesos y sustancias de partida o potenciales compuestos producidos como intermedios de reacción deben ser seleccionados de manera de prevenir accidentes químicos incluyendo escape no controlado de sustancias, explosiones e incendios.

Claramente, es imposible comentar en un espacio breve los alcances de cada uno de estos 12 postulados para dar una idea global del quehacer de la QV, su desarrollo y su vínculo con la academia y la sociedad. Para poder dar una idea clara integral el uso de ejemplos es una buena alternativa. Por esa razón, en este artículo nos enfocaremos en dos de los 12 postulados e intentaremos buscar relacionarlos con los 10 restantes de una manera coherente. Para proceder con esta ilustración tomaremos como base los postulados dos y cinco de la lista precedente. El principio de economía atómica puede ser evaluado cuantitativamente usando el modelo de Trost (Trost 1991, Trost 1998, Trost 2002):

\section{Síntesis del óxido de etileno}

Usaremos la síntesis de óxido de etileno para ilustrar cómo de un modo sencillo es posible anticipar el carácter verde o sucio de un determinado proceso. El índice de economía atómica se calcula de un modo extremadamente sencillo haciendo un count del número de átomos que intervienen como materiales de partida y todos los átomos que aparecen en el proceso completo (Trost 1991, Trost 1998, Trost 2002). Esta relación atómica se expresa en términos de las respectivas masas molares (MM) normalizadas al 100\%. El porcentaje de economía atómica (\%EA) se calcula como:

$$
\% E A=\frac{\text { MM atomos utilizados }}{\text { MM todos los reactantes }} \chi 100
$$


La ruta actualmente usada para la obtención del óxido de etileno es la que se muestra en el Esquema 1.

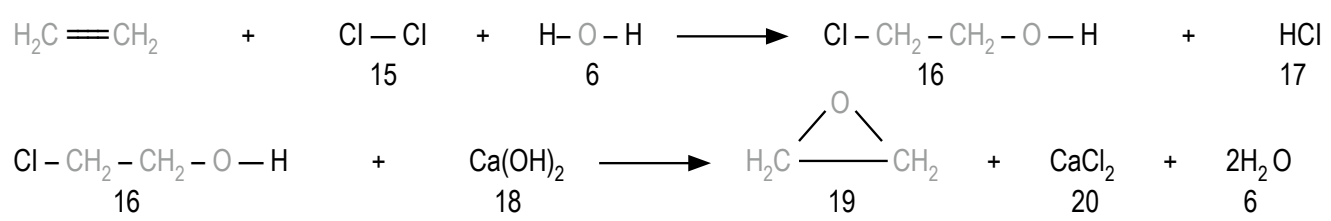

Esquema 1. Síntesis clásica del óxido de etileno (compuesto 19 en el esquema)

Rendimiento $=81 \%$ (criterio únicamente económico de eficiencia).

Índice de economía atómica: (criterio gris del proceso).

$\% E A=\frac{\text { MM atomos utilizados }}{\text { MM todos los reactantes }} \times 100$

\begin{tabular}{llllll} 
Formula Reactantes & $\begin{array}{l}\text { M.M } \\
\text { Reactantes }\end{array}$ & $\begin{array}{l}\text { Atomos } \\
\text { Utilizados }\end{array}$ & $\begin{array}{l}\text { MM de Atomos } \\
\text { Utilizados }\end{array}$ & $\begin{array}{l}\text { Atomos no } \\
\text { Utilizados }\end{array}$ & $\begin{array}{l}\text { MM de Atomos no } \\
\text { Utilizados }\end{array}$ \\
\hline $14 \mathrm{C}_{2} \mathrm{H}_{4}$ & 28 & $2 \mathrm{C}, 4 \mathrm{H}$ & 28 & - & 0 \\
\hline $15 \mathrm{Cl}_{2}$ & 71 & - & 0 & $2 \mathrm{Cl}$ & 71 \\
\hline $6 \mathrm{H}_{2} \mathrm{O}$ & 18 & 0 & 16 & $2 \mathrm{H}$ & 2 \\
\hline $18 \mathrm{Ca}(\mathrm{OH})_{2}$ & 72 & & 0 & $\mathrm{Ca}, 4 \mathrm{H}, 2 \mathrm{O}$ & 72 \\
\hline $\begin{array}{l}\text { Total } \\
2 \mathrm{C}, 8 \mathrm{H}, 30, \mathrm{Ca}, 2 \mathrm{Cl}\end{array}$ & 189 & $2 \mathrm{C}, 4 \mathrm{H}, \mathrm{O}$ & 44 & $6 \mathrm{H}, 2 \mathrm{O}, \mathrm{Ca}, 2 \mathrm{Cl}$ & 145 \\
\hline
\end{tabular}

Para ilustrar el cálculo del índice \%EA se observa que la MM de átomos utilizados es 44 respecto de la MM de reactantes es 189, de modo que el índice \%EA es:

$$
\% E A=\frac{44}{189} \times 100=23 \%
$$

Este porcentaje es manifiestamente bajo debido a la cantidad de desechos que el proceso sintético produce. El óxido de etileno es un compuesto de alta demanda en la industria chilena, con variadas aplicaciones. Es pertinente aquí preguntarse acerca del impacto que tendría eliminarlo directamente. Sin embargo, su existencia tiene claros beneficios en la industria de las soldaduras en materiales blandos, de modo que tiene claramente un beneficio innegable. De acuerdo a la ecuación (1), el índice 
de economía atómica (EA) se define como la razón entre la masa molar (MM) de los átomos utilizados para producir el compuesto deseado 19 en el Esquema 1 y la masa molar de todos los átomos que se generan en la síntesis, incluyendo intermedios persistentes, normalizados a 100\%. La ruta sintética conocida como la ruta de la cloridrina tiene una eficiencia de $81 \%$, que desde un punto de vista puramente económico es un proceso altamente eficiente. Sin embargo, el balance de $\mathrm{EA}(\% \mathrm{EA}=23 \%)$ revela que este proceso es ambientalmente ineficiente y la reacción puede catalogarse como un proceso altamente sucio desde el punto de vista del impacto ambiental. En vez de desechar la producción del compuesto 19, se puede alternativamente diseñar una ruta alternativa que conduzca al mismo producto con un índice \%EA superior. Esta ruta alternativa introduce el uso de un catalizador metálico que se muestra en el Esquema 2.

* Vea más ejemplos que ilustran el postulado de economía atómica en el sitio: http://www.chemsoc.org/pdf/gcn/atomeff.ppt
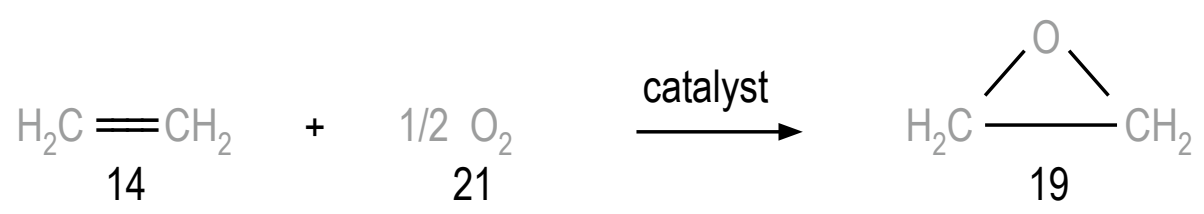

Esquema 2. Síntesis verde de óxido de etileno vía catálisis heterogénea.

Rendimiento $=70 \%$ (criterio únicamente económico de eficiencia).

\begin{tabular}{llllll} 
Formula Reactantes & $\begin{array}{l}\text { M.M } \\
\text { Reactantes }\end{array}$ & $\begin{array}{l}\text { Atomos } \\
\text { Utilizados }\end{array}$ & $\begin{array}{l}\text { MM de Atomos } \\
\text { Utilizados }\end{array}$ & $\begin{array}{l}\text { Atomos no } \\
\text { Utilizados }\end{array}$ & $\begin{array}{l}\text { MM de Atomos no } \\
\text { Utilizados }\end{array}$ \\
\hline $14 \mathrm{C}_{2} \mathrm{H}_{4}$ & 28 & $2 \mathrm{C}, 4 \mathrm{H}$ & 28 & 0 & 0 \\
\hline $211 / 2 \mathrm{O}_{2}$ & 16 & 0 & 16 & 0 & 0 \\
\hline $\begin{array}{l}\text { Total } \\
2 \mathrm{C}, 4 \mathrm{H}, 1 \mathrm{OI}\end{array}$ & 44 & $2 \mathrm{C}, 4 \mathrm{H}, 0$ & 44 & 0 & 0 \\
\hline
\end{tabular}

$$
\begin{aligned}
& \% E A=\frac{44}{44} \times 100=100 \% \\
& F E=0
\end{aligned}
$$

Note que esta vez el factor EA es ideal y la reacción es ambientalmente limpia con un factor de impacto al entorno (FE) nulo. Es también pertinente notar que a pesar 
de la impresionante diferencia desde el punto de vista de impacto ambiental, es también pertinente enfatizar que desde el punto de vista únicamente económico hay una reducción significativa de un $11 \%$ en eficiencia. Este factor no es menor, pues de acuerdo a la realidad social empírica, un empresario difícilmente se inclinaría por la ruta catalizada pues en términos puramente económicos esto se traduciría en una utilidad significativamente menor. Finalmente se puede concluir que en esta ilustración están implícitamente involucrados los postulados 1, 2, 6, 8, 9, y 10.

La diferencia en los rendimientos puramente económicos (81 vs. 70) \% no debe, desde el punto de vista de la QV, considerarse como una calamidad. Por el contrario, la esencia de la QV sugiere tomar esta aparente calamidad como un desafío: debe haber una ruta sintética alternativa que mantenga el índice EA tan alto como se pueda y que minimice la diferencia entre eficiencia únicamente económica. La pregunta contiene la solución en sí misma: se puede reemplazar el catalizador para mantener el índice EA en su valor más alto realizando una catálisis homogénea en un medio de reacción que a) sea no tóxico (Postulados 1,2, 3, 4, 8 y 10); b) que tenga propiedades catalíticas a presión y temperatura ambiente (Postulado 9). Note que según la ruta del esquema 2 el catalizador es persistente (normalmente es un catalizador metálico) y difícilmente reciclable (Postulados 5, 6, 9 y 10).

La respuesta a esta interrogante es felizmente afirmativa: los líquidos iónicos (LI). (Wasserscheid \& Welton, 2008). Un líquido iónico es esencialmente una sal fundida. De hecho, la sal común (cloruro de sodio) es un LI por sobre los $800^{\circ} \mathrm{C}$. Claramente, a esta temperatura todas las consideración acerca de un proceso verde fracasan principalmente por el factor energético. Existen dos causas principales que explican que el cloruro de sodio $(\mathrm{NaCl})$ sea sólido a temperatura ambiente, a saber, el tamaño pequeño de los iones $\mathrm{Na}+$ y $\mathrm{Cl}$ - y la alta simetría que tiene este cristal. La respuesta inmediata entonces es obtener una sal que esté compuesta por iones de gran tamaño y que posean una simetría muy baja, de modo que las interacciones entre los iones sean moderadamente débiles debido a que están a una distancia mayor, y que la baja simetría no permita un empaquetamiento fácil para dar una fase cristalina: el resultado de esta consideración es que se puede tener una sal fundida a temperatura ambiente. Este material así descrito es la definición de un líquido iónico. Este resultado es conocido desde larga data. Sin embargo, recién al inicio de la década de los noventa fue refundado, dando origen a una nueva generación de solventes con ventajas notables. Estas son las siguientes: debido a que aún en las condiciones de tamaño y simetría desfavorables estos materiales están compuestos por iones (generalmente no asociados) y como resultado tienen una presión de vapor indetectable, no son tóxicos por inhalación, al contrario de los solventes convencionales (orgánicos), muchos de los cuales tienen una demostrada actividad cancerígena o mutagénica. La segunda propiedad (y probablemente la 
más potente) es su alta flexibilidad combinatoria: considerando todos los aniones y cationes actualmente existentes, se pueden formar del orden de 1012 combinaciones como potenciales líquidos iónicos. Esta propiedad garantiza que seguramente el LI necesario para optimizar un proceso determinado se encuentra en este formidable número de combinaciones. El problema es encontrarlo. Es en este punto que la teoría ayuda y explica la razón por la cual la QV requiere de una integración teoría + experimento.

\section{LA QUÍMICA VERDE EN CHILE}

En esta sección explicaremos la actividad en QV desarrollada en el país a lo largo de sus ejes de impacto principales.

\subsection{Formación integrada en QV en niveles de pregrado y posgrado}

El grupo de Química Teórica de la Facultad de Ciencias inició un proyecto financiado por la Iniciativa Científica Milenio del ministerio de Economía y obtuvo financiamiento en 2012 por un período de tres años, renovados por otros tres años actualmente, asociando laboratorios nacionales de la Universidad Católica de Chile, la Universidad de Santiago, la Universidad Federico Santa María, la Universidad de Tarapacá y la Universidad del Desarrollo. La iniciativa, denominada Centro Interdisciplinario de Líquidos Iónicos (CILIS: www.cilis.cl), ha formado a alrededor de 70 estudiantes de pregrado, 30 estudiantes de posgrado y un número creciente de jóvenes investigadores. Estos jóvenes recibieron y reciben una formación integrada teoría + experimento en temas definidos en el ámbito de la QV. Se trata de una cifra significativa para el país.

\subsection{La QV en el colegio}

Sin duda por su naturaleza, la QV despliega su mayor impacto cuando sus conceptos y postulados son introducidos en etapas tempranas de la vida escolar. Se han utilizado dos formatos de preferencia. El primero es la interacción directa con los niños de la etapa básica y media, a través de talleres especializados adaptados a los programas definidos por el ministerio de Educación. En el período transcurrido desde 2012, aproximadamente 1500 estudiantes de diferentes niveles han participado en estos talleres o recibido charlas especializadas en la Región Metropolitana y otras regiones del centro-sur del país. La segunda estrategia, sin duda más potente por su efecto amplificador, es el perfeccionamiento de 18 profesores de enseñanza 
media de colegios seleccionados por la fundación Belén Educa. Estos docentes recibieron una formación teórica y experimental en la Facultad de Ciencias sobre los postulados e impacto en la sociedad de la QV. Esta actividad fue reconocida por el ministerio de Educación como perfeccionamiento docente y tiene un impacto en alrededor de 700 niños anualmente. Además recibió el premio UNESCO 2014 en la mención Innovación en la Enseñanza de las Ciencias. Esta actividad continuará por los próximos tres años. Paralelo a esta actividad educacional, se desarrollará durante 2015 otra actividad relacionada con el programa "Huertas urbanas", dirigido a diferentes ONG y profesores de enseñanza básica y media.

\subsection{La QV en el sector productivo nacional}

La actividad orientada al sector productivo se ha desarrollado en torno al sector minero y agrícola. El primero de ellos se desarrolló en asociación con la empresa Coppex SpA, para quienes se diseñaron y ensayaron una serie de LI para ser utilizados como materiales portadores de calor en plantas de fundición de cobre. La segunda iniciativa, en desarrollo actualmente, es el diseño de LI como aditivos mejoradores de carga para fumigación selectiva, en asociación con la empresa Tiber y co-financiada por un proyecto Corfo-Innova.

\section{CONCLUSIONES}

Este artículo ha intentado describir el estado actual de la química moderna, que introduce dentro de sus variables físicas naturales una más compleja, a saber, el impacto ambiental. Dicho de una manera técnica, las diferentes ecuaciones de estado escritas en términos de sus variables de estado naturales están siendo reemplazadas por un formalismo un poco más complejo, pero de un impacto a nivel mundial, en cuanto compromete una serie de problemas dramáticos que enfrenta la sociedad moderna. Se ha descrito cómo, de una manera sencilla, se puede realizar el cálculo de la eficiencia de un proceso químico en términos de la generación de productos deseados y consumidos por la sociedad. Esto no puede ni debe depender solo del rendimiento económico y las utilidades comerciales del mismo, sino que debe estar responsablemente relacionado con el impacto ambiental. Este cambio, en la perspectiva de procesos sostenibles, está recibiendo una atención creciente en las sociedades desarrolladas. A nivel mundial, la Química Verde está dejando de ser un objeto académico delicado y elegante para convertirse en un modo de desarrollo que resuelve el dilema entre lo puramente económico y lo puramente "ecológico". Por ejemplo, los flujos de materiales y el ahorro de energía en el largo plazo son 
claramente más rentables que una economía depredadora e irresponsable con el entorno. Hemos descrito cuál es el rol de la academia y la sociedad con relación a la conservación de los recursos naturales. Dicha descripción enfatiza el rol de la educación especializada en las universidades chilenas y la educación temprana en la escuela, orientada a formar personas conscientes de la interacción sociedadnaturaleza y responsables. Hemos ilustrado con dos ejemplos cómo la Química Verde está comenzando a ser considerada por pequeñas empresas en el país como una alternativa para soluciones de problemas específicos en las áreas de minería y agricultura. Una gran señal, sin duda.

\section{REFERENCIAS}

[1] Anastas, P. T.; Warner, J. C. Green Chemistry: Theory and Practice, Oxford University Press: New York, 1998, p.30.

[2] Cann, M. C.; Connelly, M. E. Real World Cases in Green Chemistry, American Chemical Society: Washington, DC, 2000. Ver también: the University of Scranton's Greening Across the Curriculum Web Site, http://academic. scranton.edu/faculty/CANNM1/organicmodule.html

[3] Trost, B. M. The atom economy: a search for synthetic efficiency, Science, 254: 14711477, 1991.

[4] Trost, B. M. en The Presidential Green Chemistry Challenge Awards Program: Summary of the 1998 Award Entries and Recipients; EPA744-R-98-001, U.S. Environmental Protection Agency, Office of Pollution Prevention and Toxics: Washington, DC, 1998; p 2.

[5] Trost, B. M. On investigating reactions for atom economy, Acc. Chem. Res., 35: 695705, 2002.

[6] Wasserscheid, P; Welton, T. Ionic Liquids in Synthesis. Second Edition, Wiley-VHC Verlag, 2008. 